\title{
Epidemiological Characteristics of Imported COVID- 19 Cases, March to December 2020, Chengdu, China: Implication for Quarantine and Isolation of International Travelers
}

\section{Wenqiang Zhang \\ Sichuan University \\ Yong Yue}

Chengdu Center for Disease Control and Prevention

Min $\mathrm{Hu}$

Chengdu Center for Disease Control and Prevention

Changhui Du

Chengdu Center for Disease Control and Prevention

Cheng Wang

Chengdu Center for Disease Control and Prevention

Xiaoli Tuo

Chengdu Center for Disease Control and Prevention

Xiaoman Jiang

Chengdu Center for Disease Control and Prevention

\section{Shuangfeng Fan}

Chengdu Center for Disease Control and Prevention

\section{Zhenhua Chen}

Chengdu Center for Disease Control and Prevention

Heng Chen

Chengdu Center for Disease Control and Prevention

Xian Liang

Chengdu Center for Disease Control and Prevention

Rongsheng Luan ( $\nabla$ luan_rs@scu.edu.cn )

Sichuan University

\section{Research Article}

Keywords: COVID-19, Quarantine, Travel Medicine, Epidemiologic Study Characteristics

Posted Date: November 2nd, 2021 
DOI: https://doi.org/10.21203/rs.3.rs-815516/v2

License: (c) (1) This work is licensed under a Creative Commons Attribution 4.0 International License. Read Full License 


\section{Abstract}

International flights have accelerated the global spread of Coronavirus Disease 2019 (COVID-19). Determination of the optimal quarantine period for international travelers is crucial to prevent the local spread caused by imported COVID-19 cases. We performed a retrospective epidemiological study using 491 imported COVID-19 cases in Chengdu, China, to describe the characteristic of the cases and estimate the time from arrival to confirmation for international travelers using nonparametric survival methods. Among the 491 imported COVID-19 cases, 194 (39.5\%) were asymptomatic infections. The mean age was 35.6 years $(S D=12.1$ years) and $83.3 \%$ were men. The majority $(74.1 \%)$ were screened positive for SARS-CoV-2, conducted by Chengdu Customs District, the People's Republic of China. Asymptomatic cases were younger than presymptomatic or symptomatic cases $(P<0.01)$. The daily number of imported COVID-19 cases displayed jagged changes. 95\% of COVID-19 cases were confirmed by PT-PCR within 14 days (95\% Cl: 13-15) after arriving in Chengdu. A 14-day quarantine measure can ensure non-infection among international travelers with a $95 \%$ probability. Policymakers may consider an extension of the quarantine period to minimize the negative consequences of the COVID-19 confinement and prevent the international spread of COVID-19. Nevertheless, the government should consider the balance between COVID-19 and socioeconomic development, which may cause more serious social and health crises.

\section{Introduction}

Coronavirus Disease 2019 (COVID-19), caused by the severe acute respiratory syndrome coronavirus 2 (SARS-CoV-2), has affected hundreds of millions of people and caused millions of deaths globally ${ }^{1}$. International flights provide the opportunity for the global spread of COVID-19². In Africa, air traffic is significantly associated with COVID-19 morbidity and mortality, and plays an important role in the geographical spread of the disease ${ }^{3}$. Travel restriction and travel quarantine have helped mitigate the international spread of COVID-192,4,5.

The quarantine duration was determined by the incubation period, the time from exposure to an infectious disease to the onset of symptoms. Theoretically, it's preferable to quarantine international travelers for a longer period than the maximum incubation period since the person-to-person transmission prior to symptom onset ${ }^{6-9}$. The estimated length of the incubation period of COVID-19 varies across the disease severity, model methods, and sample sizes ${ }^{10-21}$. Thus, the optimal quarantine duration for COVID-19 needs to be assessed using the previously reported incubation period.

Furthermore, a quarantine period varies all over the world. It is unclear whether the 14-day quarantine is sufficient to protect against the international spread of COVID-19. In this study, we provide direct estimates of the optimal quarantine period for international travelers, based on the interval between the date of arrival and the date of confirmation of imported COVID-19 cases in Chengdu, China. We also evaluate the effectiveness of Customs screening at detecting international travelers infected with SARSCoV-2. 


\section{Results}

\section{Demographic characteristics of COVID-19 cases}

Among the 491 imported COVID-19 cases (Table 1), 194 cases were asymptomatic (39.5\%), 114 cases were presymptomatic $(23.2 \%), 183$ cases were symptomatic (37.3\%). The mean age of the cases was 35.6 years $(S D=12.1) .409$ cases were men $(83.3 \%), 82$ cases were women $(16.7 \%)$. Most cases were workers $(189,38.5 \%)$, from Asia $(266,54.2 \%)$. The majority $(364,74.1 \%)$ of cases were screened positive for SARS-CoV-2, conducted by Chengdu Customs District, the People's Republic of China. Compared with presymptomatic or symptomatic cases, asymptomatic cases had a lower mean age $(P<0.01)$. There was no sex or occupation difference across different stages of infections $(P>0.05)$, whereas an imported continent difference was observed ( $P=0.007$, Fisher's exact test). The proportion of cases screened positive by Customs quarantine was slightly different $(P=0.043)$, while pairwise comparisons between the stages of infections showed no statistical difference $(P>0.05)$. 
Table 1

Demographic characteristics of imported COVID-19 cases in Chengdu, China

\begin{tabular}{|c|c|c|c|c|c|}
\hline & $\begin{array}{l}\text { Total } \\
(\mathrm{N}=491)\end{array}$ & $\begin{array}{l}\text { Asymptomatic } \\
(\mathrm{N}=194)\end{array}$ & $\begin{array}{l}\text { Presymptomatic } \\
(\mathrm{N}=114)\end{array}$ & $\begin{array}{l}\text { Symptomatic } \\
(\mathrm{N}=183)\end{array}$ & $\begin{array}{l}P \\
\text { value }\end{array}$ \\
\hline Age, years & $35.6 \pm 12.1$ & $32.3 \pm 10.9+$ & $36.9 \pm 13.2$ & $38.3 \pm 11.7$ & $<0.001$ \\
\hline Sex & & & & & 0.054 \\
\hline Men & $\begin{array}{l}409 \\
(83.3)\end{array}$ & $152(78.4)$ & 100 (87.7) & 157 (85.8) & \\
\hline Women & $82(16.7)$ & $42(21.6)$ & $14(12.3)$ & $26(14.2)$ & \\
\hline Occupation & & & & & 0.215 \\
\hline Worker & $\begin{array}{l}189 \\
(38.5)\end{array}$ & $72(37.1)$ & $42(36.8)$ & 75 (41.0) & \\
\hline Cadre or staff & 65 (13.2) & $29(14.9)$ & $16(14.0)$ & $20(10.9)$ & \\
\hline Student or teacher & $63(12.8)$ & $27(13.9)$ & $9(7.9)$ & $27(14.8)$ & \\
\hline Commercial service & $62(12.6)$ & $27(13.9)$ & $16(14.0)$ & 19 (10.4) & \\
\hline $\begin{array}{l}\text { Housework or } \\
\text { unemployment }\end{array}$ & $40(8.1)$ & $16(8.2)$ & $9(7.9)$ & $15(8.2)$ & \\
\hline Catering service & $34(6.9)$ & $15(7.7)$ & $6(5.3)$ & $13(7.1)$ & \\
\hline Other & $38(7.7)$ & $8(4.1)$ & $16(14.0)$ & $14(7.7)$ & \\
\hline Imported continent & & & & & $0.007 \ddagger$ \\
\hline Asia & $\begin{array}{l}266 \\
(54.2)\end{array}$ & 124 (63.9) & $59(51.8)$ & $83(45.4)$ & \\
\hline Africa & $\begin{array}{l}166 \\
(33.8)\end{array}$ & $50(25.8)$ & $45(39.5)$ & $71(38.8)$ & \\
\hline Europe & $38(7.7)$ & $15(7.7)$ & $6(5.3)$ & $17(9.3)$ & \\
\hline Americas & $21(4.3)$ & $5(2.6)$ & $4(3.5)$ & $12(6.6)$ & \\
\hline Customs quarantine & & & & & 0.043 \\
\hline Positive & $\begin{array}{l}364 \\
(74.1)\end{array}$ & 150 (77.3) & $90(78.9)$ & 124 (67.8) & \\
\hline
\end{tabular}

Data are mean \pm standard deviation or $\mathrm{n}(\%)$.

tCompared with presymptomatic or symptomatic cases, $P<0.01$, adjusted using the Bonferroni correction.

‡Fisher's exact test. 


\begin{tabular}{|c|c|c|c|c|c|}
\hline & $\begin{array}{l}\text { Total } \\
(\mathrm{N}=491)\end{array}$ & $\begin{array}{l}\text { Asymptomatic } \\
(\mathrm{N}=194)\end{array}$ & $\begin{array}{l}\text { Presymptomatic } \\
(\mathrm{N}=114)\end{array}$ & $\begin{array}{l}\text { Symptomatic } \\
(\mathrm{N}=183)\end{array}$ & $\begin{array}{l}P \\
\text { value }\end{array}$ \\
\hline Negative & $\begin{array}{l}127 \\
(25.9)\end{array}$ & $44(22.7)$ & $24(21.1)$ & $59(32.2)$ & \\
\hline \multicolumn{6}{|c|}{ Data are mean \pm standard deviation or $\mathrm{n}(\%)$} \\
\hline \multicolumn{6}{|c|}{$\begin{array}{l}\text { †Compared with presymptomatic or symptomatic cases, } P<0.01 \text {, adjusted using the Bonferroni } \\
\text { correction. }\end{array}$} \\
\hline ‡Fisher's exact test. & & & & & \\
\hline
\end{tabular}

\section{Epidemic Curve}

Progression of the imported COVID-19 epidemic in Chengdu correlated with airline control measures implemented by the Civil Aviation Administration of China. Flight plans adjustment that each foreign airline would be only allowed to maintain one route to China with no more than one weekly flight was implemented as of March 29, 2020. International routines decreased, and imported COVID-19 cases decreased. Incentives and circuit breaker measures for flights have been implemented since June 8, 2020. International travelers increased, and imported COVID-19 cases increased. The international route would be suspended, once the circuit breaker was triggered. The number of imported COVID-19 cases demonstrated jagged changes (Figure 1).

\section{Time to confirmation}

The cumulative distribution of time to confirmation, defined as the time between the date of arriving in Chengdu and the date of confirmation of COVID-19 by PT-PCR, is shown in Figure 2. Among 491 cases, 95\% were confirmed by PT-PCR within 14 days (95\% Cl: 13-15) after arriving in Chengdu. For different stages of infections (Figure 3 A), 95\% of asymptomatic cases were confirmed by PT-PCR within 15 days (13-16) after arrival, $95 \%$ of presymptomatic cases were confirmed by PT-PCR within 13 days (13-13) after arrival, $95 \%$ of symptomatic cases were confirmed by PT-PCR within 14 days (13-17) after arrival. Furthermore, $95 \%$ of male cases were confirmed by PT-PCR within 14 days (13-15) after arrival, $95 \%$ of female cases were confirmed by PT-PCR within 14 days (12-16) after arrival (Figure 3B).

\section{Discussion}

This analysis of imported COVID-19 cases in Chengdu, China, provides insight into formulating a rationale for determining the optimal quarantine period for international travelers. The values estimated provide the evidentiary foundation for containing the international spread of COVID-19. Analyses of the interval between the date of arrival and the date of confirmation of imported COVID-19 cases revealed that at least $5 \%$ of incubation periods could be longer than 14 days. These results paint a positive picture 
of the impact of obligatory household quarantine for 14 days in Chengdu. Public health authorities may consider a slight extension of the quarantine period to deal with uncertainty in the incubation periods.

All ages of the population are susceptible to SARS-CoV-2 infection, younger adults who contracted SARSCoV-2 are more likely to be asymptomatic or have mild diseases ${ }^{22-24}$. We found that asymptomatic cases were younger than presymptomatic or symptomatic cases. The age-dependent T-cell and B-cell dysfunction and differential expression of inflammatory genes could lead to immune dysregulation and induce more prolonged proinflammatory responses, potentially contributing to poor outcomes ${ }^{25-27}$.

This work further supports a high prevalence of asymptomatic cases in SARS-CoV-2 infections ${ }^{28,29}$. $39.5 \%$ of international travelers infected with SARS-CoV-2 remains asymptomatic, which is lower than the range reported for SARS-CoV-2 $(40 \%-45 \%)^{29}$. Asymptomatic international travelers will be missed by symptom-based surveillance ${ }^{30}$, even if screened for SARS-CoV-2, so it is crucial to implement the travel quarantine for international travelers during the COVID-19 pandemic.

Our estimation of the 95th quantile of the interval between the date of arrival and the date of confirmation, 14 days, supports the adopted mandatory quarantine period of 14 days in China ${ }^{19}$. Of note, we estimated that about $5.0 \%$ of imported COVID-19 cases would not have a positive SARS-CoV-2 RT-PCR array until 14 days after arriving in Chengdu. Similarly, previous work had suggested that at least $5 \%$ of cases would take 14 days or more to develop symptoms ${ }^{19-21}$. In particular, asymptomatic cases can transmit SARS-CoV-2 to others for longer than 14 days $^{29}$. It has appeared several COVID-19 outbreaks in China due to international imported COVID-19 cases $^{31}$. Consequently, the 14-day household quarantine was recommended to international travelers discharged from a designated centralized

quarantine site for 14 days in Chengdu.

Our study has several limitations. First, international travelers arriving in Chengdu might be a biased sample due to the airline control measures implemented by the Civil Aviation Administration of China. Second, we could not estimate the incubation period distribution of imported COVID-19 cases since the possible exposure time of imported COVID-19 cases was not available. Moreover, a minority of international travelers who arrived in December 2020 received COVID-19 vaccines, so we could not evaluate the impact of the vaccines. Although vaccination is effective and safe against COVID-1932,33, the vaccinated population may contract SARS-CoV-2 and infect others ${ }^{34-36}$. Finally, we could not assess the true transmissibility of imported cases with an extended incubation period, longer than 14 days. A longitudinal study should be carried out to investigate the infectious period of COVID-19 or how long infected individuals remain infectious to others, which is associated with release from quarantine.

\section{Conclusions}

The high prevalence of asymptomatic infection and the long incubation period have made it challenging to contain the COVID-19 pandemic. To ensure non-infection with a 95\% probability, public health 
authorities should quarantine international travelers for more than 14 days. Also, policymakers may consider an obligatory household quarantine or household health monitoring after a 14-day designated centralized quarantine, to minimize the negative consequences of the COVID-19 confinement and prevent the international spread of COVID-19. Nevertheless, the government should consider the balance between COVID-19 and socioeconomic development, which may cause more serious social and health crises.

\section{Methods}

\section{Source of data}

A retrospective observational study of the imported laboratory-confirmed COVID-19 cases from March 2020 to December 2020 was performed. All international travelers, arriving in Chengdu, should be screened for SARS-CoV-2, shortly conducted by Chengdu Customs District, the People's Republic of China. Confirmations of COVID-19 cases were done at the Chengdu Center for Disease Control and Prevention, using reverse transcription polymerase chain reaction (RT-PCR) assays targeting two different regions of the RdRp gene in SARS-CoV-2. All methods were carried out in accordance with relevant guidelines. All experiments were carried out in the Laboratory of Chengdu Center for Disease Control and Prevention. Regardless of symptoms, all international travelers should comply with a centralized isolated medical quarantine for 14 days and household quarantine for 14 days in Chengdu. Basic demographic information and time from arrival to confirmation by RT-PCR were recorded for all confirmed cases. We excluded 2 imported COVID-19 cases who received a centralized isolated medical quarantine in the other city, subsequently arrived in Chengdu, and were confirmed by RT-PCR. Hence, we included 491 cases in the study.

\section{Case definitions}

COVID-19 cases were divided into asymptomatic, presymptomatic, and symptomatic cases. Asymptomatic cases were defined as being confirmed by RT-PCR without any symptoms. Presymptomatic cases were defined as being confirmed by RT-PCR, prior to the onset of symptoms. Symptomatic cases were defined as being confirmed by RT-PCR and the development of symptoms.

\section{Time to confirmation}

In this study, "time to confirmation" was defined as the interval in days between the date of arriving in Chengdu and the date of confirmation of COVID-19 by PT-PCR.

\section{Statistical analysis}


Age was present as mean with standard deviation (SD), which was subject to normal distribution. We performed the Shapiro-Wilk test of normality and computed Levene's test for homogeneity of variance across the stages of infections. In this study, the variance was tested to be not equal. We used an approximate method of Welch ${ }^{37}$ to compare age according to the stages of infections, which generalizes the commonly known 2-sample Welch test to the case of arbitrarily many samples. Pairwise comparisons between the stages of infections were calculated with the Bonferroni corrections for multiple testing. Sex, occupation, imported continent, Customs quarantine were presented as absolute numbers with the percentage. Chi-square tests and Fisher's exact tests were used to compare the difference according to the stages of infections. Time between arrival and confirmation was estimated by the use of nonparametric survival methods. The 95th quantile of time to be confirmation and corresponding $95 \%$ confidence interval $(C l)$ were computed. All analyses were performed using $\mathrm{R}$ version 4.0.4 (R Foundation for Statistical Computing).

\section{Declarations}

\section{Authors' contributions}

W.Z. conceptualized the study, conducted statistical analyses, and drafted the manuscript. Y.Y., M.H., C.D., C.W., X.T., X.J., S.F., Z.C., and H.C., collected data and discuss analysis. X.L. and R.L. advised on the interpretation of results and were responsible for the study. All authors approved the final manuscript.

\section{Conflict of interest}

The authors declare no conflict of interest.

\section{Funding}

This research was funded by the Chengdu Municipal Science and Technology Bureau Key R\&D Support Program Technology Innovation R\&D Project (2020-YF05-00037-SN, 2020-YF05-00133-SN), the Non-profit Central Research Institute Fund of Chinese Academy of Medical Sciences (2020-PT330-005), Sichuan Medical Science and Technology Project (21ZDCX003), Sichuan Science and Technology Program (2021YFS0001).

\section{Role of sponsors}

The sponsors had no role in the design of the study, the analysis of the data, or the preparation of the manuscript.

\section{Ethical Approval}


Data collection was determined by the Article 7, 12, 48, Law of the People's Republic of China on Prevention and Treatment of Infectious Diseases to be part of the epidemiological investigation of a notifiable infectious disease and therefore the individual informed consent was waived. Partial data was available from the official website of Chengdu Municipal Health Commission (http://cdwjw.chengdu.gov.cn/cdwjw/c135632/yqbd.shtml).

Paragraph 1 of Article 7: Center for Disease Control and Prevention at all levels shall undertake infectious disease monitoring, prediction, epidemiological investigation, epidemic reporting, and other prevention and control work.

Paragraph 1 of Article 12: All units and individuals within the territory of the people's Republic of China must accept the investigation, inspection, sample collection, isolation treatment and other prevention and control measures associated with infectious diseases by Center for Disease Control and Prevention and medical institutions, and truthfully provide relevant information. Center for Disease Control and Prevention and medical institutions shall not disclose relevant information and materials involving personal privacy.

Paragraph 1 of Article 48: When an epidemic of infectious diseases occurs, Center for Disease Control and Prevention and other professional and technical institutions related to infectious diseases designated by the Administrative Department of Health of the People's Government at or above the provincial level may enter the epidemic focus and epidemic area of infectious diseases for investigation, sample collection, technical analysis and testing.

\section{Data Availability Statements}

All data are available in the supplementary material. All codes are available by emailing Wenqiang Zhang (zwqscu@126.com) on reasonable request.

\section{Acknowledgements}

The authors are grateful to all the field staff and participants for their contributions.

\section{References}

1 World Health Organization. WHO Coronavirus (COVID-19) Dashboard, <https://covid19.who.int> (2021).

2 Wells, C. R. et al. Impact of international travel and border control measures on the global spread of the novel 2019 coronavirus outbreak. Proc Natl Acad Sci U S A117, 7504-7509, doi:10.1073/pnas.2002616117 (2020). 
3 Osayomi, T. et al. A Geographical Analysis of the African COVID-19 Paradox: Putting the Poverty-as-aVaccine Hypothesis to the Test. Earth Systems and Environment, doi:10.1007/s41748-021-00234-5 (2021).

4 Chinazzi, M. et al. The effect of travel restrictions on the spread of the 2019 novel coronavirus (COVID19) outbreak. Science368, 395-400, doi:10.1126/science.aba9757 (2020).

5 Costantino, V., Heslop, D. J. \& Maclntyre, C. R. The effectiveness of full and partial travel bans against COVID-19 spread in Australia for travellers from China during and after the epidemic peak in China. Journal of travel medicine27, doi:10.1093/jtm/taaa081 (2020).

6 Gao, Y. et al. A cluster of the Corona Virus Disease 2019 caused by incubation period transmission in Wuxi, China. The Journal of infection80, 666-670, doi:10.1016/j.jinf.2020.03.042 (2020).

7 Tindale, L. C. et al. Evidence for transmission of COVID-19 prior to symptom onset. eLife9, doi:10.7554/eLife.57149 (2020).

8 Yu, P., Zhu, J., Zhang, Z. \& Han, Y. A Familial Cluster of Infection Associated With the 2019 Novel Coronavirus Indicating Possible Person-to-Person Transmission During the Incubation Period. J Infect Dis221, 1757-1761, doi:10.1093/infdis/jiaa077 (2020).

9 Johansson, M. A. et al. SARS-CoV-2 Transmission From People Without COVID-19 Symptoms. JAMA network open4, e2035057, doi:10.1001/jamanetworkopen.2020.35057 (2021).

$10 \mathrm{Nie}, \mathrm{X}$. et al. Epidemiological Characteristics and Incubation Period of 7015 Confirmed Cases With Coronavirus Disease 2019 Outside Hubei Province in China. J Infect Dis222, 26-33, doi:10.1093/infdis/jiaa211 (2020).

11 Qin, J. et al. Estimation of incubation period distribution of COVID-19 using disease onset forward time: A novel cross-sectional and forward follow-up study. Science advances6, eabc1202, doi:10.1126/sciadv.abc1202 (2020).

12 Lauer, S. A. et al. The Incubation Period of Coronavirus Disease 2019 (COVID-19) From Publicly Reported Confirmed Cases: Estimation and Application. Annals of internal medicine172, 577-582, doi:10.7326/M20-0504 (2020).

13 Gussow, A. B., Auslander, N., Wolf, Y. I. \& Koonin, E. V. Prediction of the incubation period for COVID-19 and future virus disease outbreaks. BMC Biol.18, 186, doi:10.1186/s12915-020-00919-9 (2020).

$14 \mathrm{Lai}, \mathrm{C}$. et al. Shorter incubation period is associated with severe disease progression in patients with COVID-19. Virulence11, 1443-1452, doi:10.1080/21505594.2020.1836894 (2020).

15 McAloon, C. et al. Incubation period of COVID-19: a rapid systematic review and meta-analysis of observational research. BMJ Open10, e039652, doi:10.1136/bmjopen-2020-039652 (2020). 
16 Backer, J. A., Klinkenberg, D. \& Wallinga, J. Incubation period of 2019 novel coronavirus (2019-nCoV) infections among travellers from Wuhan, China, 20-28 January 2020. Euro surveillance : bulletin Europeen sur les maladies transmissibles = European communicable disease bulletin25, doi:10.2807/1560-7917.ES.2020.25.5.2000062 (2020).

$17 \mathrm{Li}, \mathrm{Q}$. et al. Early Transmission Dynamics in Wuhan, China, of Novel Coronavirus-Infected Pneumonia. The New England journal of medicine382, 1199-1207, doi:10.1056/NEJMoa2001316 (2020).

$18 \mathrm{He}, \mathrm{W} ., \mathrm{Yi}, \mathrm{G}$. Y. \& Zhu, Y. Estimation of the basic reproduction number, average incubation time, asymptomatic infection rate, and case fatality rate for COVID-19: Meta-analysis and sensitivity analysis. J Med Viro/92, 2543-2550, doi:10.1002/jmv.26041 (2020).

19 Lu, Q. B. et al. Epidemiological parameters of COVID-19 and its implication for infectivity among patients in China, 1 January to 11 February 2020. Euro surveillance : bulletin Europeen sur les maladies transmissibles $=$ European communicable disease bulletin25, doi:10.2807/15607917.ES.2020.25.40.2000250 (2020).

20 Lai, C. K. C. et al. Epidemiological characteristics of the first 100 cases of coronavirus disease 2019 (COVID-19) in Hong Kong Special Administrative Region, China, a city with a stringent containment policy. International journal of epidemiology49, 1096-1105, doi:10.1093/ije/dyaa106 (2020).

$21 \mathrm{Bi}$, Q. et al. Epidemiology and transmission of COVID-19 in 391 cases and 1286 of their close contacts in Shenzhen, China: a retrospective cohort study. The Lancet Infectious Diseases20, 911-919, doi:10.1016/s1473-3099(20)30287-5 (2020).

22 Hu, B., Guo, H., Zhou, P. \& Shi, Z. L. Characteristics of SARS-CoV-2 and COVID-19. Nat. Rev. Microbiol.19, 141-154, doi:10.1038/s41579-020-00459-7 (2021).

23 Guan, W. J. et al. Clinical Characteristics of Coronavirus Disease 2019 in China. The New England journal of medicine382, 1708-1720, doi:10.1056/NEJMoa2002032 (2020).

24 The Novel Coronavirus Pneumonia Emergency Response Epidemiology Team. The Epidemiological Characteristics of an Outbreak of 2019 Novel Coronavirus Diseases (COVID-19) - China, 2020. China Cdc Weekly2, 113-122, doi:10.46234/ccdcw2020.032 (2020).

25 Zhou, F. et al. Clinical course and risk factors for mortality of adult inpatients with COVID-19 in Wuhan, China: a retrospective cohort study. The Lancet395, 1054-1062, doi:10.1016/s0140-6736(20)30566-3 (2020).

26 Smits, S. L. et al. Exacerbated innate host response to SARS-CoV in aged non-human primates. PLoS Pathog6, e1000756, doi:10.1371/journal.ppat.1000756 (2010).

27 Opal, S. M., Girard, T. D. \& Ely, E. W. The immunopathogenesis of sepsis in elderly patients. Clinical infectious diseases : an official publication of the Infectious Diseases Society of America41 Suppl 7, 
S504-512, doi:10.1086/432007 (2005).

28 Shakiba, M., Nazemipour, M., Heidarzadeh, A. \& Mansournia, M. A. Prevalence of asymptomatic COVID-19 infection using a seroepidemiological survey. Epidemiology and infection148, e300, doi:10.1017/S0950268820002745 (2020).

29 Oran, D. P. \& Topol, E. J. Prevalence of Asymptomatic SARS-CoV-2 Infection : A Narrative Review. Annals of internal medicine173, 362-367, doi:10.7326/M20-3012 (2020).

30 Quilty, B. J., Clifford, S., Flasche, S., Eggo, R. M. \& group, C. n. w. Effectiveness of airport screening at detecting travellers infected with novel coronavirus (2019-nCoV). Euro surveillance : bulletin Europeen sur les maladies transmissibles = European communicable disease bulletin25, doi:10.2807/15607917.ES.2020.25.5.2000080 (2020).

31 Testing Team, L. \& Zhang, W. Local Outbreak of COVID-19 in Shunyi District Attributed to an Asymptomatic Carrier with a History of Stay in Indonesia - Beijing Municipality, China, December 23, 2020. China Cdc Weekly3, 214-217, doi:10.46234/ccdcw2020.062 (2021).

32 Jara, A. et al. Effectiveness of an Inactivated SARS-CoV-2 Vaccine in Chile. The New England journal of medicine, doi:10.1056/NEJMoa2107715 (2021).

33 Lopez Bernal, J. et al. Effectiveness of Covid-19 Vaccines against the B.1.617.2 (Delta) Variant. The New England journal of medicine385, 585-594, doi:10.1056/NEJMoa2108891 (2021).

34 Team, C. C.-V. B. C. I. COVID-19 Vaccine Breakthrough Infections Reported to CDC - United States, January 1-April 30, 2021. MMWR. Morbidity and mortality weekly report70, 792-793, doi:10.15585/mmwr.mm7021e3 (2021).

35 Philomina, J. B. et al. Genomic survey of SARS-CoV-2 vaccine breakthrough infections in healthcare workers from Kerala, India. The Journal of infection83, 237-279, doi:10.1016/j.jinf.2021.05.018 (2021).

36 Hacisuleyman, E. et al. Vaccine Breakthrough Infections with SARS-CoV-2 Variants. The New England journal of medicine384, 2212-2218, doi:10.1056/NEJMoa2105000 (2021).

37 Welch, B. L. On the Comparison of Several Mean Values - an Alternative Approach. Biometrika38, 330336, doi:DOI 10.1093/biomet/38.3-4.330 (1951).

\section{Figures}




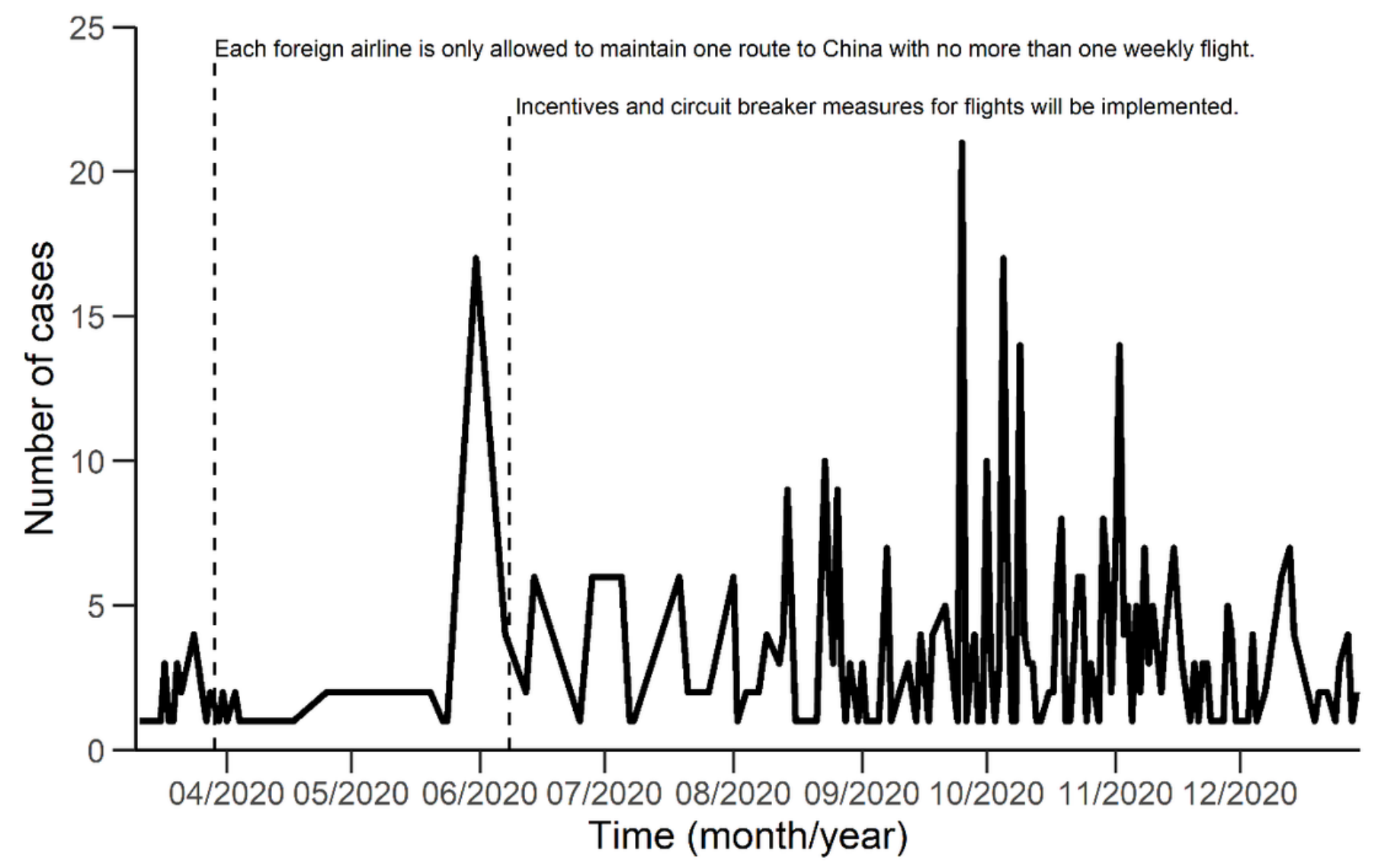

Figure 1

Trend of confirmed COVID-19 cases, March to December 2020 in Chengdu, China 


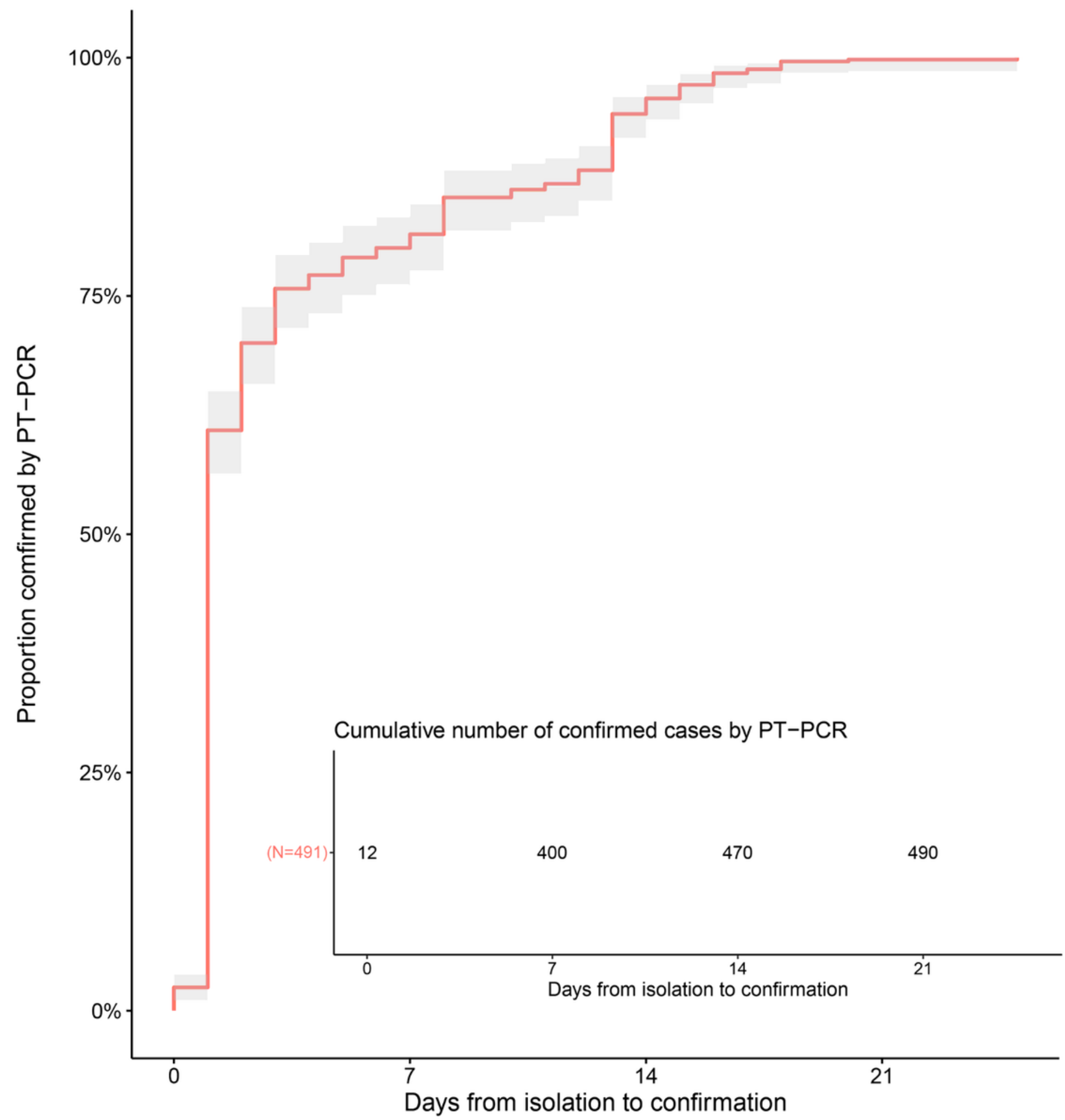

\section{Figure 2}

Time between arrival in Chengdu and SARS-CoV-2 confirmation. We estimated that $95 \%$ of cases were confirmed by PT-PCR within 14 days (95\% Cl: 13-15) after arriving in Chengdu. 

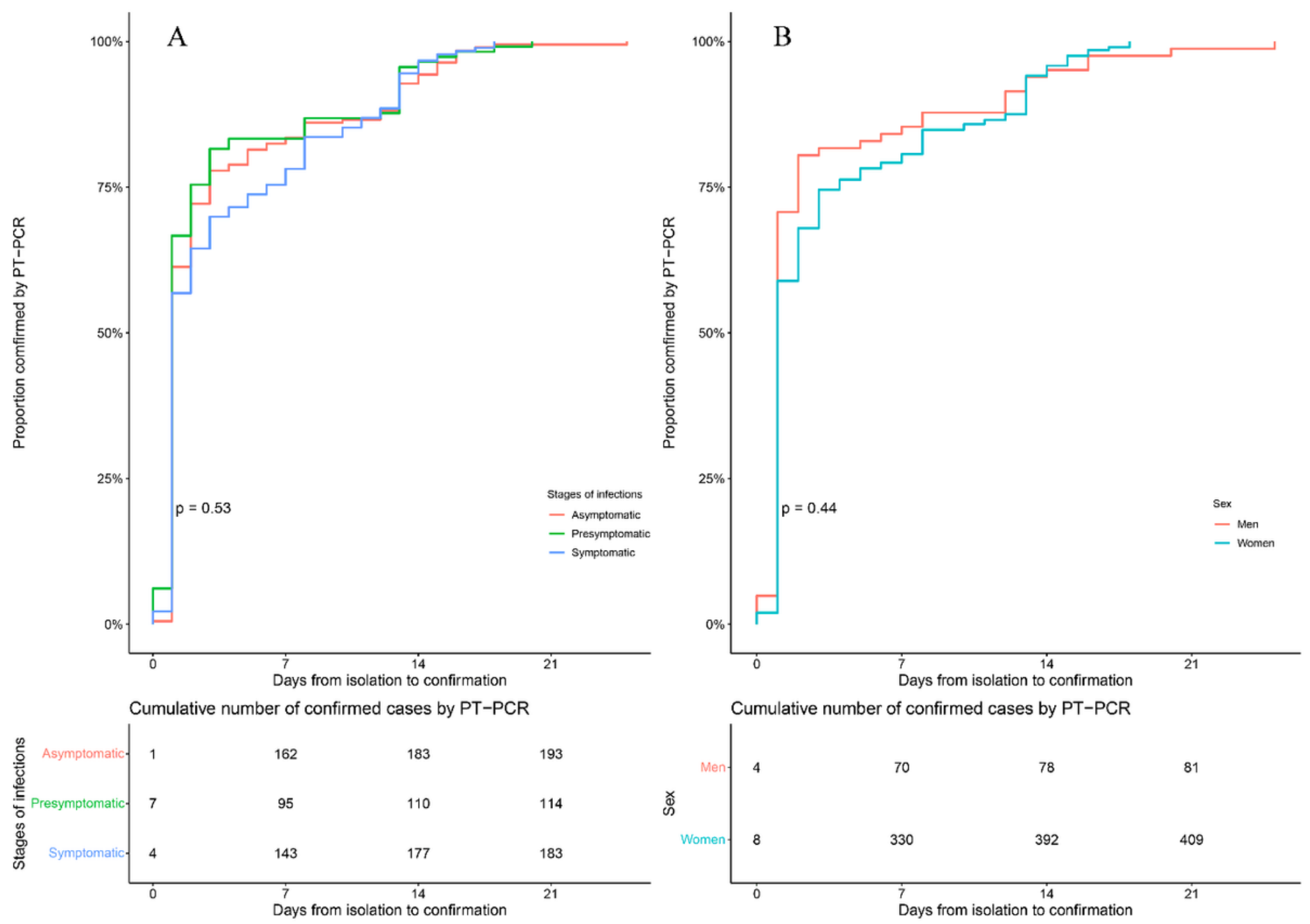

Figure 3

Time between arrival in Chengdu and SARS-CoV-2 confirmation by stages of infections (A), sex (B). Panel A shows estimates of the proportion of cases at different stages who are confirmed by PT-PCR, according to the number of days after arriving in Chengdu. We estimated that $95 \%$ of asymptomatic cases were confirmed by PT-PCR within 15 days (95\% Cl: 13-16) after arrival, $95 \%$ of presymptomatic cases were confirmed by PT-PCR within 13 days (13-13) after arrival, $95 \%$ of symptomatic cases were confirmed by PT-PCR within 14 days (13-17) after arrival. Panel B shows estimates of the proportion of cases for different sexes who are confirmed by PT-PCR, according to the number of days after arriving in Chengdu. We estimated that $95 \%$ of male cases were confirmed by PT-PCR within 14 days (95\% Cl: 13-15) after arrival, $95 \%$ of female cases were confirmed by PT-PCR within 14 days (12-16) after arrival. The upper confidence limit for female cases and presymptomatic cases are not available and estimated using the 95th quantile and corresponding lower confidence limit.

\section{Supplementary Files}

This is a list of supplementary files associated with this preprint. Click to download. 
- Data.xlsx

Page 17/17 